$$
\text { Comet } 1886 \ldots \text { (Fabry). }
$$

April r. In der hellen Abenddämmerung und bei dunstiger Luft erschien der Comet als ein heller verdichteter Nebel von 2' Durchmesser. Der Durchmesser der Verdichtung ist o'5. Lalande giebt für den Vergleichstern in Decl. 12.7 . und Weisse 5.9.

$$
\text { Comet } 1886 \ldots \text { (Barnard). }
$$

Dec. I 2. Da meine Cometenposition von Dec. 12 stark abwich, hatte ich den Vergleichstern BB.VI + $5^{\circ} .59$ I an BB.VI + $5^{\circ} 594$ angeschlossen (vergl. A. N. 2703 ). Inzwischen hat Herr Prof. Schönfeld mitgetheilt, dass die Decl. von $+5^{\circ} .594$ von Argelander nicht beobachtet, sondern nur gegen +5.595 geschätzt worden ist. In Folge dessen konnte mein Anschluss

Dresden I 886 April I 3. in Decl. nicht verwendet werden, und ich ersuchte daher Herrn Oberst Pomerantzeff in Taschkent den Stern BB.VI $+5^{\circ} .59$ I am Meridiankreise zu bestimmen, was auch bereitwilligst, obwohl bei ungünstigen atmosphärischen Verhältnissen, ausgeführt wurde. Danach hat Stern BB.VI +5.59 I die grosse jährliche Eig. Bew. von to. $165^{\circ}$ und -1."022.*) Vorstehende Beobachtung ist aus A. N. 2703 wiederholt.

März 8. Dunst. Comet mässig hell, I' Durchmesser. Verdichtung in vorangehenden Cometentheile.

» 2 . Dunst. Comet hell, $3^{\prime}$ Durchmesser. Die Verdichtung ist im vorangehenden Cometentheile.

April I. Dunst. Der Comet ist hell, mit einem fächerförmigen Schweife von 2' Länge. Kernártige Verdichtung im vorangehenden Cometentheile.

$$
\text { B. von Engelhardt. }
$$

*) Die angegebene grosse E. B. muss, so lange nicht die auf I Faden und I Mikroskop beruhende Mer. Beob. in BB.VI + $5^{\circ}$.59I anderweitig verificirt werden kann, als sehr 2weifelhaft betrachtet werden. Die Durchmusterungszone 307, beobachtet I854, giebt nach einer Mitthi ilung des Geh. Rath Schönfeld mit Taschkent übereinstimmend die Rect. $4^{\mathrm{h}} \mathbf{2}^{\mathrm{m}} 7^{\mathbf{s}} \mathbf{2}$ (1885.0), spricht also entschieden gegen die E. B.

\title{
Bemerkung zu: T. W. Backhouse, Proposed Maps for tracing Meteor-paths (A. N. 2714).
}

[Auszug aus einem Schreiben des Herrn F. Folie, Director der Sternwarte in Brüssel, an den Herausgeber.]

„L'auteur me semble attribuer une importance tout à fait exagérée, et hors de proportion avec le but à atteindre, à l'exactitude absolue de ces cartes et au nombre d'étoiles qu'elles devraient renfermer.

Pour moi, j'estime que des Cartes faites sur le plan de celles d'Al. Dorna, à une échelle un peu plus grande, seraient complètement suffisantes.

Si l'auteur veut donner suite à son idée, il pourra fort utilement consulter l'uranométrie générale. de Houzeau, et si les cartes qu'il se propose de faire sont destinées à tous les observatoires de l'Europe, qui sont situés entre $40^{\circ}$ et $60^{\circ}$ de latitude en chiffres ronds, il semble plus naturel de les faire pour l'horizon de $50^{\circ}$ que pour celui de Greenwich.

Bruxelles le 5 Avril i 886 .

F. Folie.«

\section{Aufforderung, betr. Doubletten älterer Bände und Nummern der Astronomischen Nachrichten.}

An den Unterzeichneten werden häufig Anfragen in Betreff einzelner älterer Bände und Nummern der Astr. Nachr. gerichtet, welche zur Completirung unvollständiger Exemplare dieser Zeitschrift gewünscht werden. Durch die Freundlichkeit einiger Herren Astronomen, welche mir die in ihrem Besitze befindlichen Doubletten zur Verfugung gestellt haben, bin ich häufig in der Lage gewesen, solchen Wïnschen entsprechen zu können, in manchen Fällen ist es mir aber nicht möglich gewesen, die gesuchten Nummern zu beschaffen. Unzweifelhaft befinden sich nun auf manchen

Kiel I 886 April 4.
Sternwarten und im Privatbesitze noch Doubletten aus den ersten Jahren des Erscheinens der Astr. Nachr., und ich möchte, da dieselben für die Besitzer doch nahezu werthlos sind, sie dagegen in vielen Fällen zur Completirung von grossem Nutzen sein können, durch diese Zeilen Anregung zur Sammlung solcher Doubletten geben. Ich bin gern bereit, dieselben zu erwerben, und bitte, sie mir freundlichst mit Angabe des Preises zur Verfugung zu stellen, oder eventuell dafür fehlende Nummern in Austausch zu nehmen.

C.F.W. Peters.

\section{In halt:}

Zu Nr.2724-25. Edward S. Holden. Note to the Editor relating to the 36 inch Clark objective for the Lick Observatory. I77. - G. Müller. Beobachtungen über den Einfluss der Phase auf die Lichtstärke kleiner Planeten. 177. - E. Frisby. Observations of Minor Planets. 195. - C. Schrader. Beobachtungen von Planeten am Meridiankreise der Sternwarte in Hamburg. I97. - K. G. Olsson. Bestimmung der Bahn des Cometen I88I VIII, 20I. - E. Millosevich. Osservazioni della cometa 1885 V. 205. - B. von Engelhardt. Cometenbeobachtungen. 205. - F. Folie. Bemerkung zu: T. W. Backhouse, Proposed Maps for tracing Meteor-paths (A. N. 27 I4). 207. - C.F.W. Peters. Aufforderung betr. Doubletten älterer Bände und Nummern der Astronomischen Nachrichten. 207. 